\title{
Comparative Study on Cold Resistance Physiology of Red Pulp Pitaya and White Pulp Pitaya
}

\author{
Yan Gong ${ }^{1}$, Xiaoyi Bi ${ }^{1}$, Lijun Deng ${ }^{1}$, Juan $\mathrm{Hu}^{1}$, Shan Jiang ${ }^{1}$, Lin Tan ${ }^{1}$, Tingting Wang ${ }^{1}$, and Xian Luo ${ }^{1 *}$ \\ ${ }^{1}$ College of Horticulture, Sichuan Agricultural University, Chengdu, Sichuan, China
}

\begin{abstract}
The red pulp pitaya variety 'Taiwan No. 2' and the white pulp pitaya variety 'white crystal' were used as experimental materials, which were cold stressed at low temperature of $3{ }^{\circ} \mathrm{C}$ for $48 \mathrm{~h}, 96 \mathrm{~h}$, and recovering at room temperature after $96 \mathrm{~h}$ cold stress for control. The relative conductivity (REC), malondialdehyde content (MDA), soluble sugar content (SS), soluble protein content (SP), free proline content (Pro), activity of superoxide dismutase (SOD), and catalase (CAT) were determined and the cold resistance were analyzed. The results showed that, after cold stress, the SP, Pro content and SOD and CAT activities of 'White Crystal' pitaya were higher than that of 'Taiwan No. 2' pitaya. It showed that the cold resistance of 'White Crystal' white pulp pitaya was stronger than that of 'Taiwan No. 2' red pulp pitaya.
\end{abstract}

\section{Introduction}

Hylocereus undatus is a perennial climbing fleshy plant of the cactus family, which is a typical tropical fruit originating in tropical desert areas of central and south America such as Mexico and Brazil. The flesh of pitaya fruit is rich in polyphenols, which is a good source of antioxidants. The peel of pitaya fruit can inhibit the growth of melanoma cells [1]. Pitaya fruit also has the function of absorbing $\mathrm{CO}_{2}$ at night, releasing $\mathrm{O}_{2}$ and purifying air. It is a good environmental protection plant. Therefore, pitaya fruit has high nutritional, economic, social and ecological value, and has become an excellent green health product.

The cultivation of pitaya has a long history in its origin, but its development is slow. In recent years, pitaya has been planted in other areas on a large scale. The introduction of pitaya varieties in China began in the early 1990s. At present, the planting area of pitaya in China is about 35000 hectare, but mainly concentrated in the subtropical zone of China. The planting area is narrow and restricted by climate and geographical conditions. Low temperature is the main environmental stress factor that limits the development of the pitaya planting 0 . Even in the warm subtropical region, the cultivation of pitaya may be affected by low temperature chilling or freezing damage. $0{ }^{\circ} \mathrm{C}$ is the minimum threshold that most pitaya cultivars can tolerate. When the temperature is lower than $5^{\circ} \mathrm{C}$, it may cause pitaya to be frozen. Buds, twigs, and even some mature branches can also be frozen. When the temperature is lower than $-2{ }^{\circ} \mathrm{C}$ for more than $12 \mathrm{~h}$, the pitaya plant will be seriously injured and when the temperature is lower than $-4{ }^{\circ} \mathrm{C}$, the plants will die 2 . The lower the temperature and the longer the duration, the more serious the impact on the yield and quality of the pitaya 3 . Therefore, it is very important to select and breed cold-resistant pitaya varieties.

There are three species of pitaya, namely, red peel with white pulp (Hylocereus undatus), red peel with red pulp (Hylocereus costaricensis), yellow peel with white pulp (Hylocereus megalanthus), among which former two species are common in the market. There are great differences in cold resistance among different species of pitaya.

At present, the research on cold resistance of pitaya is mainly focused on the changes of physiological indexes of different varieties of pitaya fruit with treatment time at semi-lethal temperature, and the changes of physiological indexes of tissue culture seedlings of different genotypes of pitaya fruit at low temperature. There are few reports about the differences in cold resistance among different species of pitaya. Our experiment aimed at the cold resistance of two common red and white pulp pitaya on the market. The purpose of the study is to clarify the tolerance of two kinds of pitaya to low temperature stress, select the varieties with stronger cold resistance, and provide a theoretical basis for the introduction and cultivation of pitaya in temperate regions, at the same time broaden the cultivation areas and improve production.

\section{Materials and methods}

\subsection{Materials}

Two varieties of pitaya plants (the red pulp pitaya variety 'Taiwan No. 2' and the white pulp pitaya variety 'white crystal') were purchased from the Yuerong Pitaya Base in Jinqiao Town, Shuangliu District, Chengdu City, Sichuan Province, China. 


\subsection{Experimental Design and Methods}

The pitaya branches with the same growth potential and without pests and diseases were selected and placed in the greenhouse of the fifth teaching building roof of Chengdu Campus of Sichuan Agricultural University. Three plants were planted in each pot for adaptive growth and unified management of fertilizer and water cultivation. Eighteen pots of red and white pulp pitaya branches were selected and cultured in artificial climate chamber (RXZ-500D intelligent type, Ningbo Jiangnan Instrument Factory). The illumination intensity was set at $2000 \mathrm{~lx}$, the photoperiod was $12 \mathrm{~h} \mathrm{light} / 12 \mathrm{~h}$ dark, the temperature was $25{ }^{\circ} \mathrm{C}$ (light) $/ 15{ }^{\circ} \mathrm{C}$ (dark), and the relative humidity was $70 \%-75 \%$.

After the growth of the plants were basically the same, the plants were selected for low temperature treatment. Nine pots of red and white pulp pitaya branches were transferred to a refrigerator at $3{ }^{\circ} \mathrm{C}$, respectively. The light intensity provided by the LED light source in the refrigerator was $2000 \mathrm{~lx}$, and the photoperiod was set to $12 \mathrm{~h} / 12 \mathrm{~h}$ by the timer. The pitaya's branches were sampled at $0 \mathrm{~h}$ (control, ck), $48 \mathrm{~h}, 96 \mathrm{~h}$ and $96 \mathrm{~h}$ in room temperature after $96 \mathrm{~h}$ cold stress (96R), respectively. Then, the sampling branches were washed and cut into small pieces, and measured the relative conductivity (REC), malondialdehyde content (MDA), soluble sugar content (SS), soluble protein content (SP), free proline content (Pro), activity of superoxide dismutase (SOD) and catalase (CAT).

REC was determined by referring to the improved method of Deng [4]. The SP, SS, MDA, Pro, SOD and CAT were determined by the methods of Liu [5] and Xiong [6], respectively.

\subsection{Statistic Analyses}

Statistical analyses were performed using Microsoft Excel 2016 and SPSS 22.0 statistical software. Data were analyzed by one-way ANOVA with least significant difference (LSD) at 5\% confidence level.

\section{Results and Analysis}

\subsection{Effects of Low Temperature Stress on MDA Content of Pitaya}

As shown in Fig.1.A, the change rate of MDA content in red pulp pitaya was higher than that in white pulp pitaya. In normal temperature control, the content of MDA in white pulp pitaya was higher than that in red pulp. After low temperature treatment, the content of MDA in red pulp pitaya increased continuously from $0 \mathrm{~h}$ to $96 \mathrm{~h}$, and the increase range in these stage was higher than that in white pulp, while the content of MDA in white pulp was significantly lower than that in red pulp.

After restoring to room temperature for 96 hours, the content of MAD in two species of pitaya decreased rapidly. However, red pulp species are still higher than white pulp species varieties.

\subsection{Effect of Low Temperature Stress on REC of Pitaya}

The REC of pitaya branches fluctuated with low temperature treatment, indicating that low temperature had a certain complicated effect on REC of pitaya (Fig.1.B). The REC value of the white pulp pitaya in the normal temperature control $(0 \mathrm{~h})$ was higher than that of the red pulp, and the difference was significant.

When under low temperature treatment, the REC value of red pulp pitaya was higher than that of the white pulp pitaya, indicating that the low temperature stress was more harmful to the red pulp pitaya.

After $96 \mathrm{~h}$ of low temperature recovery treatment, the REC values of the two species of pitaya were lower than the corresponding normal temperature control values, indicating that the cell membrane permeability of these two pitaya varieties could be restored to a greater extent under suitable conditions.

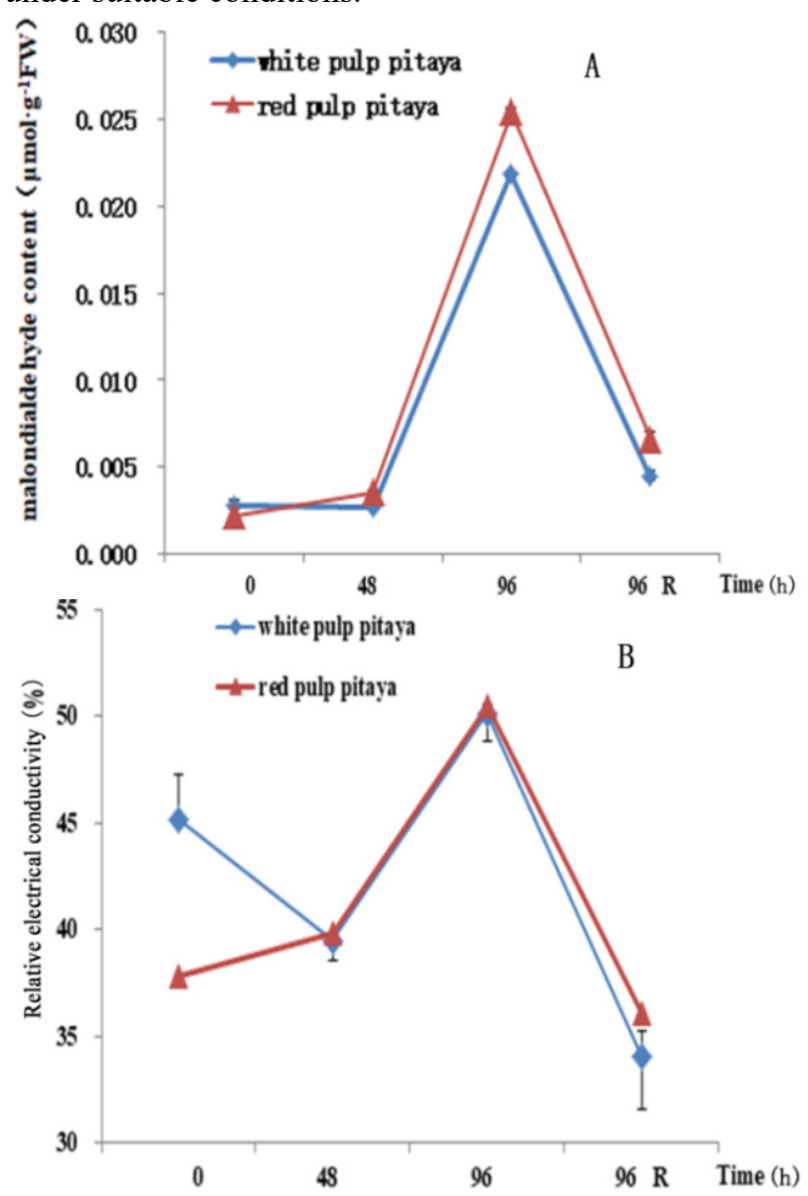

Fig. 1. Changes of MDA (A) content and REC (B) in pitaya under cold stress

Note: 'R' in the horizontal axis means restore in room temperature for 96 hours after 96 hours of cold stress. The same below.

\subsection{Effect of Low Temperature Stress on CAT Activity of Pitaya}

Hydrogen peroxide is a waste produced in the course of metabolism, which can cause damage to the cell. Plants often produce more hydrogen peroxide under low 
temperature stress. In order to avoid this damage, hydrogen peroxide must be rapidly converted into other harmless or less toxic substances. Catalase is often used by cells to catalyze the decomposition of hydrogen peroxide. As shown in Fig.2.A, the CAT activity of the two pitaya species decreased first and then increased with the increase of the low temperature treatment time. The activity of the two species of pitaya was higher than that of the normal temperature control after 96 hours. In normal temperature control and low temperature treatment, the CAT activity of white pulp pitaya was higher than that of white pulp.

\subsection{Effect of Low Temperature Stress on SOD Activity of Pitaya}

The SOD activity of both red and white pitaya increased first and then decreased with the increase of treatment time (Fig.2.B). The SOD value of white pulp pitaya in normal temperature control was higher than that of red pulp. The SOD value of red pulp in each stage of low temperature treatment was higher than that of white pulp. The SOD activity value and activity increase time and amplitude of white pulp pitaya were higher than that of red pulp after low temperature treatment.
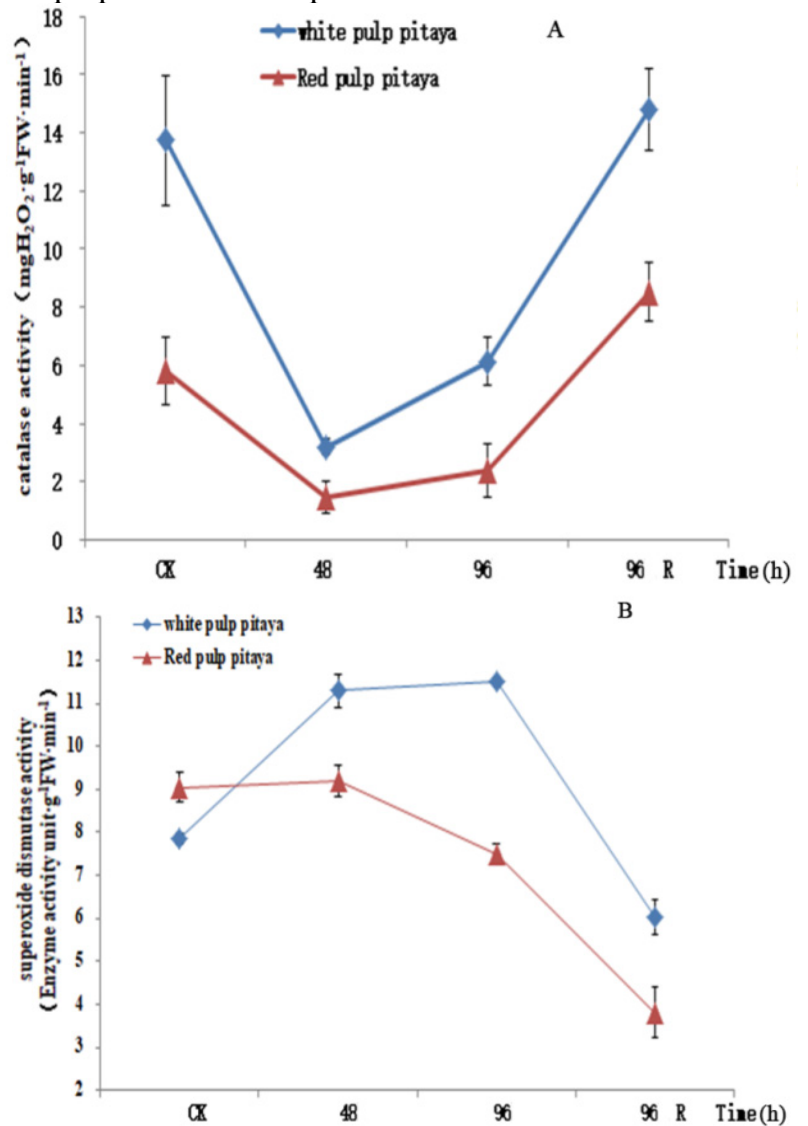

Fig. 2. Changes of CAT (A) and SOD (B) activity of pitaya under low temperature stress

\subsection{Effect of Low Temperature Stress on Proline Content of Pitaya}

Proline (Pro) is one of the components of plant proteins, and it can exist in free state widely in plants. Under the stress conditions of low temperature, drought and salinity, proline accumulated in many plants. The accumulated proline not only acts as osmotic regulator in plant cytoplasm, but also plays an important role in stabilizing biological macromolecular structure, reducing cell acidity, eliminating ammonia toxicity and regulating cell redox as an energy pool. Varieties with strong cold resistance tend to accumulate more proline. Therefore, the determination of proline content can be used as a physiological index for cold resistance breeding. In addition, proline is highly hydrophilic and can stabilize the protoplast colloid and metabolic processes in tissues, thus reducing the freezing point and preventing cell dehydration. As shown in Fig.3.A, in the normal temperature control and each low temperature treatment, the Pro content of white pulp pitaya was higher than white pulp. And the low content of white pulp pitaya Pro after low temperature treatment was higher than the normal temperature control.

\subsection{Effect of Low Temperature Stress on Soluble Sugar Content of Pitaya}

After low temperature treatment, the soluble sugar content of red pulp and white pulp pitaya showed a decreasing trend (Fig.3.B). The soluble sugar content of white pulp in each treatment stage was higher than that of red pulp.
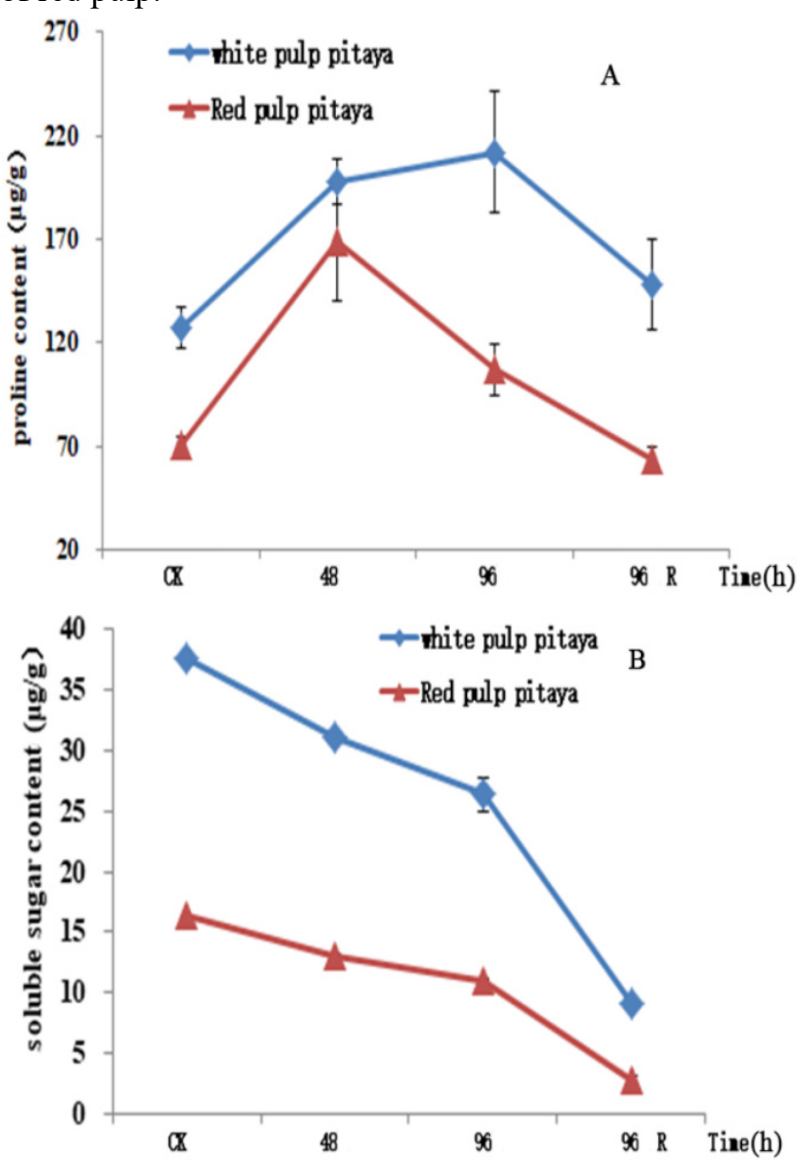

Fig. 3. Changes of proline (A) and soluble sugar content (B) in pitaya under low temperature stress 


\section{Conclusion}

The activities of Pro, SOD and CAT of 'White Crystal' pitaya branched were higher than those of 'Taiwan 2'. The results showed that the cold resistance of 'White Crystal' white pulp pitaya branches were stronger than that of 'Taiwan 2' red pulp pitaya.

\section{References:}

1. T. Liu, L. Yang, P. Ying, R. Wang, X. Shen. Anhui Agri. Sci., 44: 40-42, (2016).

2. Y. Ortiz-Hernández, J. Carrillo-Salazar. Comun. Sci. 3: 220-237, (2012).

3. R. J. Deng, J. X. Fan, Y. Q. Wang, Chinese J. Plant Physiol., 50: 1742-1748, (2014).

4. R. J. Deng, J. X. Wang, Plant Physiol. Commun., 45, 1023-1026, (2009).

5. Z. Y. Liu, S. C. Zhang, Physiol. of Plant Resistance, China Agriculture Press, Beijing, 46-51, (1994).

6. Q. Y. Xiong, Experimental Course of Plant Physiol. Sichuan Science and Technology Press, Chengdu, 66-78, (2003). 